Original paper

\title{
An overview of recent developments in FLUKA PET TOOLS
}

\author{
R.S. Augusto ${ }^{\mathrm{a}, \mathrm{b}, *}$, J. Bauer $^{\mathrm{c}}$, O. Bouhali ${ }^{\mathrm{d}}$, C. Cuccagna ${ }^{\mathrm{a}, \mathrm{e}, \mathrm{f}}$, C. Gianoli ${ }^{\mathrm{b}}$, W.S. Kozłowska ${ }^{\mathrm{a}, \mathrm{g}}$, \\ P.G. Ortega ${ }^{h}$, T. Tessonnier ${ }^{\mathrm{i}}$, Y. Toufique ${ }^{\mathrm{d}, \mathrm{j}}$, V. Vlachoudis ${ }^{\mathrm{a}}$, K. Parodi ${ }^{\mathrm{b}}$, A. Ferrari ${ }^{\mathrm{a}}$ \\ ${ }^{\text {a } C E R N ~-~ E u r o p e a n ~ O r g a n i z a t i o n ~ f o r ~ N u c l e a r ~ R e s e a r c h, ~ C H-1211 ~ G e n e ̀ v e ~ 23, ~ S w i t z e r l a n d ~}$ \\ ${ }^{\mathrm{b}}$ Ludwig-Maximilians-Universität München, Am Coulombwall 1, 85748 Garching, Germany \\ ${ }^{\mathrm{c}}$ UniversitätsKlinikum Heidelberg, Heidelberger Ionenstrahl-Therapiezentrum HIT, Germany \\ d Texas A\&M University at Qatar, 23874 Doha, Qatar \\ e TERA Foundation, Via Puccini 11, 28100 Novara, Italy \\ ${ }^{\mathrm{f}}$ Université de Genève, 30 quai Ernest-Ansermet, CH-1211 Genève, Switzerland \\ ${ }^{8}$ Medizinische Universität Wien, Spitalgasse 23, 1090 Vienna, Austria \\ ${ }^{\text {h }}$ Grupo de Física Nuclear, Universidad de Salamanca, E-37008 Salamanca, Spain \\ ${ }^{\mathrm{i}}$ Centre François Baclesse, 3 Avenue du Général Harris, 14000 Caen, France \\ ${ }^{\mathrm{j}}$ Institut Superieur des Sciences de la Santé de Settat, Morocco
}

\section{A R T I C L E I N F O}

\section{MSC:}

00-01

99-00

Keywords:

Monte Carlo

FLUKA

PET

\begin{abstract}
A B S T R A C T
The new developments of the FLUKA Positron-Emission-Tomography (PET) tools are detailed. FLUKA is a fully integrated Monte Carlo (MC) particle transport code, used for an extended range of applications, including Medical Physics. Recently, it provided the medical community with dedicated simulation tools for clinical applications, including the PET simulation package. PET is a well-established imaging technique in nuclear medicine, and a promising method for clinical in vivo treatment verification in hadrontherapy. The application of clinically established PET scanners to new irradiation environments such as hadrontherapy requires further experimental and theoretical research to which MC simulations could be applied. The FLUKA PET TOoLs, besides featuring PET scanner models in its library, allow the configuration of new PET prototypes via the FLUKA Graphical User Interface (GUI) Flair. Both the beam time structure and scan time can be specified by the user, reproducing PET acquisitions in time, in a particle therapy scenario. Furthermore, different scoring routines allow the analysis of single and coincident events, and identification of parent isotopes generating annihilation events. Two reconstruction codes are currently supported: the Filtered Back-Projection (FBP) and Maximum-Likelihood Expectation Maximization (MLEM), the latter embedded in the tools. Compatibility with other reconstruction frameworks is also possible. The FLUKA PET TooLs package has been successfully tested for different detectors and scenarios, including conventional functional PET applications and in beam PET, either using radioactive sources, or simulating hadron beam irradiations. The results obtained so far confirm the FLUKA PET TOoLs suitability to perform PET simulations in R\&D environment.
\end{abstract}

\section{Introduction}

\subsection{Positron emission tomography}

PET designates a medical imaging technique characterized by the indirect detection of $\beta^{+}$emitting radionuclides, through the detection of coincidence photons from annihilation events. This technique is now clinically well-established and characterized by detectors with relatively high efficiency and resolution. The reconstructed images are able to highlight metabolic functions of the tissue (functional imaging), as well as repercussions of irradiation in treatment verification scenarios, both in 4D [1]. Since its origins, in the 70's, it underwent many conceptual changes, leading to the modern PET design and technical specifications $[2,3]$. However, the main principle is still maintained: detector rings process the coincidence data from annihilation photons, considering the events acquired within a narrow energy and time coincidence window, scored throughout its Field of View (FOV).

In nuclear medicine the common use of PET is diagnostics, requiring the injection of a tracer in the patient's body, for example FDG (fluorodeoxyglucose), a glucose molecule coupled with the $\beta^{+}$emitting isotope ${ }^{18} \mathrm{~F}$, employed to evaluate the glucose uptake of the body cells to search for any dysfunction. Besides its conventional use in nuclear medicine, PET is presently one of the most promising techniques for radiotherapy treatment monitoring and range verification in the

\footnotetext{
* Corresponding author at: CERN - European Organization for Nuclear Research, CH-1211 Genève 23, Switzerland.

E-mail address: r.s.augusto@cern.ch (R.S. Augusto).
} 
framework of hadrontherapy [4]. In this PET application, no radioisotope injection is required as the $\beta^{+}$activity is generated as a result of fragmentation mechanisms (auto activation) [5]. The charged hadron beam dose distribution in a target corresponds to a Bragg Peak, which in turn can be detected via correlation with the ensuing $\beta^{+}$emitter distribution verified through PET. However, such correlation between activity and dose is complex, requiring models to estimate the magnitude and spatial distribution of those fragments based on the beam and patient geometry characteristics [6-8]. Reconstructed PET images are therefore of the utmost importance in order to correlate the activity density of $\beta^{+}$emitter nuclei to the dose, and particularly to the range. This is the most explored way to obtain information on irradiation since no primary beam particle exits the body, although other promising methods such as prompt $\gamma$ or other secondary particles detection are being studied [9-15]. Notwithstanding this, $\beta^{+}$activity can be more directly induced in a target, by using radioactive ion $\beta^{+}$emitting beams and thus mitigating the correlation errors [16-19].

A typical reconstruction process involves the representation of sinograms - a 2D projection in space representing a transaxial slice, with one dimension allocated for the radial distance from the center and the other representing the projection angle - and transverse slice images in a cylindrical region of interest limited by the axial FOV as denoted in Fig. 1. Special algorithms for reconstruction of tomographic images can be applied to obtain a $\beta^{+}$emitter distribution, acting over either 2D or 3D data. The latter includes more information, as it evaluates crossed coincidences among detector rings, although eventually the images are generally converted into $2 \mathrm{D}$ projections. They usually require either the application of inverse Fourier transform to get the data into an image matrix (Filtered Back Projection - FBP) or iterative algorithms converging to the $\beta^{+}$activity distribution using generally multiple corrective factors, often scanner-specific to mitigate noise influence and increase spatial resolution (Maximum Likelihood Expectation Maximization - MLEM). The latter is a more sophisticated and efficient method, involving maximizing the logarithm of a Poisson-likelihood function, but can be rather time-consuming to converge [3].

\subsection{Rationale behind FLUKA PET TOOLS}

PET tools $[20,21]$ are one of the major medical-themed features currently being developed in FLUKA [22-24], along with the HADRONTHERAPY platform which includes several utilities of relevance to hadrontherapy applications. These packages aim at extending FLUKA's functionalities from high energy physics into the medical domain. In parallel, the FLUKA's Graphical User Interface (GUI) Flair [25] has undergone significant improvements in treatment planning quality assurance recalculations and optimization. In fact, all these upgrades are part of a continuous effort to provide improved models and features in the code for medical applications throughout recent years [24]. The

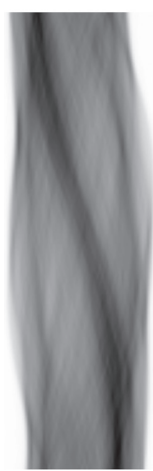

Sinogram

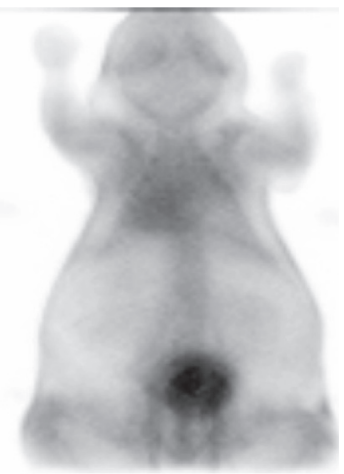

Projection View
PET TOoLs package aims at exploiting the full predictive potential of FLUKA for different purposes, including: the reproduction of the PET/ CT scanner response for diagnostics imaging; the development of new scanners; the validation of treatment scenarios (dosimetry) and range monitoring in hadrontherapy. In particular, PET Tooss can be used to assess:

- Coincidence types and imaging quality, according to experimental conditions (e.g. geometry, equipment, patient anatomy). Also, the effects of different acquisition times on image quality can be tested.

- Performance of innovative materials (e.g. scintillation materials) based on their detection capabilities (stopping power, high $\mathrm{Z}$ and density), signal output studies (in energy and time) and low noise propensity.

- Signal to noise ratio, estimating the scanner optimized count rate.

- Efficient time resolution, as the acquisition time may change in the view of the annihilation position or due to scintillator decay time not being instantaneous. Effects of different pulse time, dead-time and coincidence timing window can be tested to estimate random events impact.

- Optimal energy window, to maximize scanner performance.

- Spatial resolution of the scanner setup, which is influenced by the positron range and the annihilation photons non-collinearity [26].

Both the imaging quality and count rate performance will be affected by the characteristics (position, size, efficiency) of the detector, the geometry of the object of interest (patient or sample) and, for hadrontherapy, the underlying physics of interactions with matter governing the production of $\beta^{+}$emitters. Due to FLUKA's reproduction of nuclear interactions and $\beta^{+}$emitter production, it can be used to estimate experimental outcomes, which is particularly relevant in R\&D stages. Thus, the figure of merit in a PET scanner test will be chiefly assessed by how well the detected $\beta^{+}$distribution compares to the simulated one [6].

Still, PET/CT applications in treatment planning for hadrontherapy, and subsequently refinement of the treatment approach (in vivo beam range verification and treatment confirmation $[27,28]$ ) can particularly benefit from this package. Since the correlation between signal and dose is not straightforward for stable ions, as it results from the interplay of multiple physical processes, it relies heavily on models to assess the distribution of $\beta^{+}$emitters' activity in time. This distribution can now be scored with FLUKA's annihilation events at rest estimator [29]. Ideally, a virtual PET reconstruction could be compared to a real reconstruction to benchmark and strengthen the treatment plan in some specific cases.

An interesting research scenario is in vivo range verification for carbon ion therapy with in beam PET or online PET $[28,30]$. This technique benefits from the availability of short-lived $\beta^{+}$emitters, mitigating the lack of signal statistics and the biological washout effect on imaging [6,31]. PET tools could be used to simulate online PET verification scenarios, testing various setup configurations. For instance, in such scenarios, detector rings have to be either opened or shifted so that the beam can pass through the ring on its way to the patient [32-34]. As a consequence, prompt radiation noise and the effect of reconstruction artifacts are non-negligible [35]. The high customization cost of such solutions is one of the main reasons which limits the clinical use of PET in vivo range verification with charged particles to offline images obtained a posteriori $[36,37,31]$. The development of dedicated dual-head PET scanners such as BASTEI [38,39] has shown the feasibility of online PET [40,35], and in addition more recent experimental data acquired with DoPET [41] and INSIDE [42,43] in beam PET detectors, have demonstrated the possibility to introduce this technique in the clinical routine.

Fig. 1. Sinogram representation of a mouse and corresponding projection view. 


\section{Materials and methods}

\subsection{Tools description}

Now embedded into FLUKA, PET TOoLs provide images reconstructed from the PET signal generated by a predetermined radioactive source or beam irradiation as acquired in a modeled PET scanner. Moreover, it provides also simulation data, which can be accessed and analyzed by the user. These tasks can now be accomplished with minimum coding via a set of prepared tools, with documentation to support the user throughout the entire PET simulation process. One example of synergies between interfaced features is the possibility of creating FLUKA-wise patient voxelized geometries for simulations. Based on the imported DICOM [44] CT scans, Flair translates Hounsfield Units (HU) using the Schneider parametrization [45] into tissue materials with an accurate density and composition description to be used for realistic PET simulations. Moreover, with the upgraded Flair hadrontherapy platform, it is now possible to simulate the entire particle treatment scenario based on the beam data from DICOM-RT Treatment Planning files. These features can be also combined with the PET scoring.

The workflow is summarized in Fig. 2 and consists of a set of tools acting sequentially (blue colored) in conformity with a FLUKA simulation, whose elements are colored in shades of green. Firstly, the PET scanner can be assembled either via the geometry editor environment in Flair or directly in the FLUKA input, these geometry tools will be explained afterwards in more detail. The scanner structures created are then interpreted by the scoring routines as detector elements for the signals generated throughout the simulation, according to the radiation source and geometry defined. After the simulation, a general output (USERDUMP) is printed, listing various details of the interactions with the scanner which can be analyzed by the user. The postprocessing routines can then be employed to convert the general output information into a rearranged list of coincidences and eventually into sinograms, in subsets of true, random and scattering coincidences. Presently, this still requires a set of parameters to be parsed via an external text file (postproc.param) with a predefined list of arguments which can be changed by the user, some examples and a full explanation of these arguments are provided in the tools documentation. In addition to that, the PET Tools feature an embedded MLEM code for image reconstruction, which may be applied to the coincidence lists, although the user can resort to external tools for that purpose, as will be demonstrated later in this work.

\subsubsection{Radiation sources}

PET tools support all types of sources that can be generated in FLUKA, such as: generic radioactive sources of various geometrical shapes for the calibration of PET detectors $\left(e . g .{ }^{68} \mathrm{Ge},{ }^{22} \mathrm{Na}\right)$; activity maps sources for functional PET simulations (e.g. ${ }^{18} \mathrm{~F}$ maps) and also beams. The $\beta^{+}$emitter distribution simulated can then be evaluated as in PET monitoring scenarios in hadrontherapy. The user can set the beam time structure irradiation information in the simulation process and acquisition time interval(s) either $a$ priori in the input, or modify the output data at post-processing stage. The latter option is more flexible for a posteriori data analysis, as the dataset is already generated. Therefore, it was the selected course of action for the in beam PET scenarios described in the presented work.

\subsubsection{PET geometry tools}

These tools allow the generation of different PET scanners with reproducible geometrical elements and their roto-translation. The user can select from (some) existing commercial models or define his/her own with customized materials/geometry. As detailed in [24], the construction of the scanner takes place in three steps of increasing complexity: blocks $\rightarrow$ modules $\rightarrow$ rings in a Flair environment as depicted in Fig. 3.

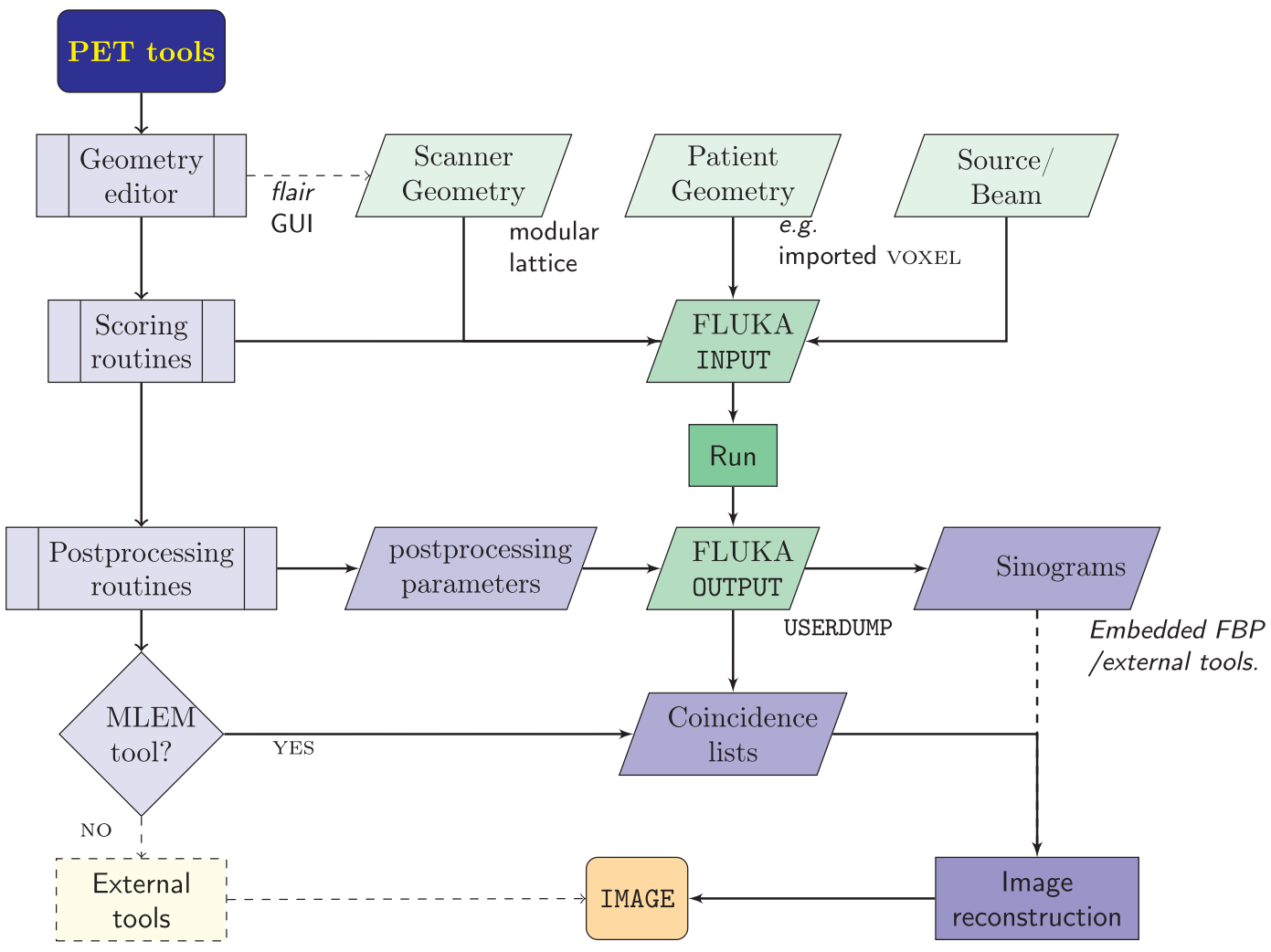

Fig. 2. FLUKA PET TOoLs workflow throughout simulation setup up to the final image reconstruction. The dashed lines denote optional features whereas the solid lined functions are automatically handled by the tools. The USERDUMP corresponds to a built-in FLUKA estimator including the necessary output (in ASCII format) from the simulation, which can be processed for the coincidence events information and ultimately image reconstruction. 


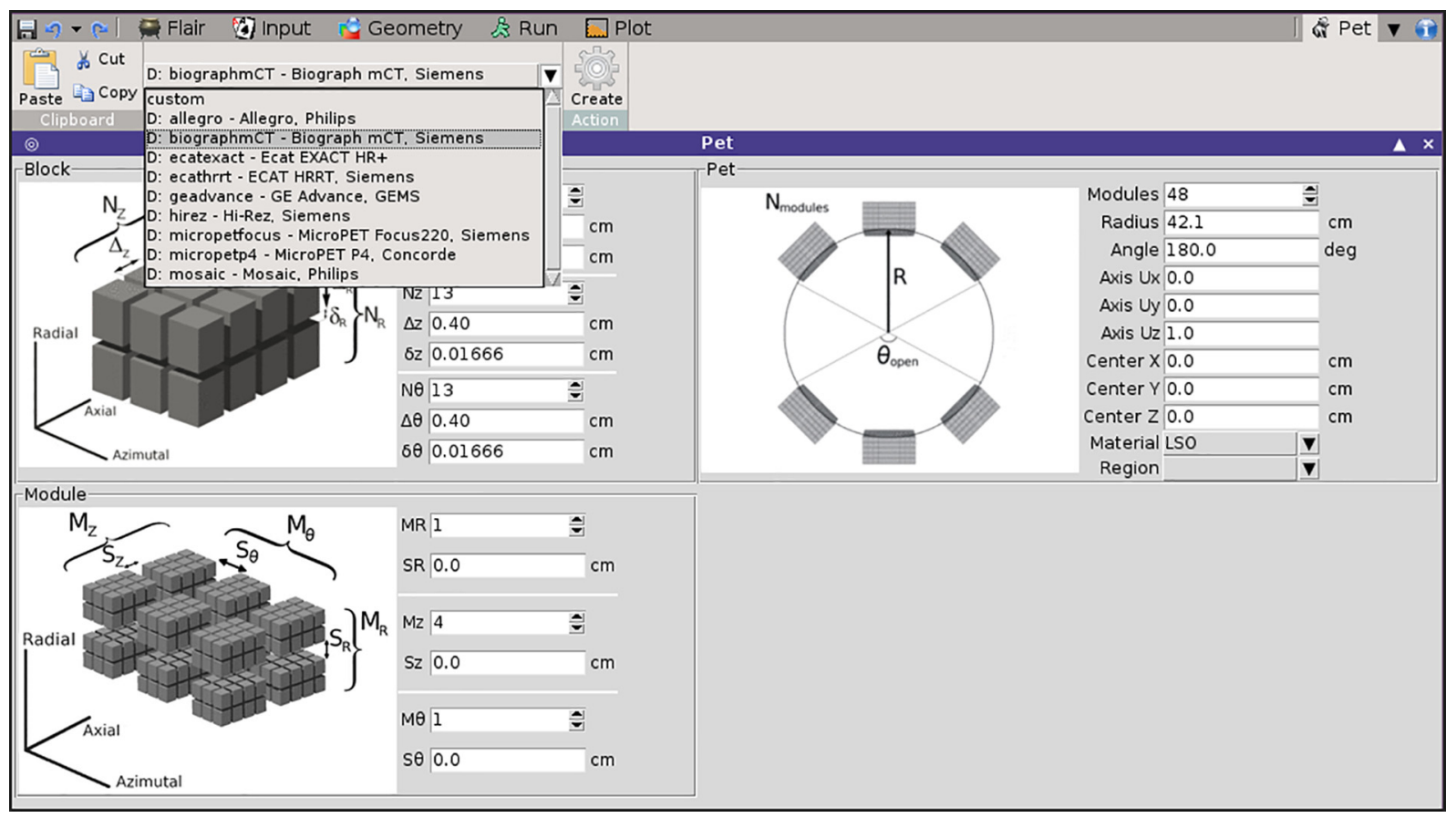

Fig. 3. Flair tab with PET geometry setup options and scanner models options displayed [24].

\subsubsection{Scoring routines}

All the simulation data, relevant to the reconstruction (e.g. scanner hit position and energy deposited information) is collected by these routines and associated with the detector geometry, in either binary or Ascil file format. The user can activate flags for the collection of additional information at the expense of data size increase (e.g. parent isotope flags). Dead time and energy window constraints are enforced through input parameters.

\subsubsection{Post-processing routines}

These consist of routines embedded in the FLUKA code and the external file to parse detector information to the routines. These allow for processing of the general output files, and the creation of both a coincidence list or sinogram representation, from the LOR and coincidence timing window. In both cases, the total coincidences are produced, along with files discriminating among scatter, random and true coincidences.

For each pair of crystal rings (user-defined), a sinogram, storing data in either 2D or 3D in a binary file, will be created. In order to apply iterative reconstruction algorithms to 3D data, the data are first rebinned into two dimensional sinograms employing Fourier rebinning. This methodology results in relatively fast reconstructions, but may incur resolution losses. Therefore, the characteristics and quality of these sinograms can be improved with several post-processing parameters, such as: Arc correction, Maximum ring difference, Number of segments, Span and Mashing factor [20,24].

The Arc correction accounts for the cylindrical shape of the PET scanner in the reconstruction and the Maximum ring difference controls the extent of the crossed coincidences by modulating the allowed difference of rings involved in the reconstruction. Number of segments, relevant for 3D reconstruction, divides the Michelogram [46] into sectors according to the different angles between those rings. The Span is a parameter pertaining the extension of segments in the Michelogram. As for the Mashing factor, it refers to the rebinning of sinogram data to reduce its dimensions [24].

\subsubsection{Imaging tools}

Using the embedded iterative MLEM based reconstruction algorithm [47], the coincidence list can be transformed into an image. The distributed tools also include an FBP reconstruction code that can be applied to the sinogram files. Although this produces lower quality images, it is much faster and preferable for quickly checking the results. External reconstruction algorithms can be applied to both the coincidence list or sinogram for further optimization. To ease the manipulation and visualization of coincidence information with an external software, the sinogram output data are stored as Interfile 3.3 files, a standardized format for nuclear medicine image data files [48], which ensures compatibility with external processing algorithms. In fact, a variety of external free software can be used to read and visualize the produced files (e.g. STIR [49], AMIDE [50]) and various reconstruction procedures and/or corrections can be applied to it. Please note that, despite the lack of attenuation corrections in the tool, the time-of-flight and resolution modeling is handled at post-processing level and the user can still apply corrective techniques on the output data. However, work is ongoing to improve the imaging tools with attenuation correction for use with MLEM reconstruction.

Scanner model compliance with technical/geometry specifications should be ensured by the user. A manual and some templates, including commercial PET scanner models and a few popular examples with instructions, are provided within the tools' package. A user can also include the noise distribution, replicating the background (e.g. by means of a radiation source) in the simulation. However, in the event of a fully integrated in beam PET simulation, the effect of neutrons and other prompt secondaries on the noise levels will already be accounted for in time according to the beam time structure and acquisition time parameters chosen by the user.

\section{Results}

\subsection{Geometry visualization}

Provided a PET device is set up properly, with an appropriate patient voXEL or phantom geometry, a source or in beam PET scenario can be simulated. This involves the reproduction of the beam time structure and acquisition time in the tools' post-processing routines. Regarding non-irradiation scenarios, a simple source with multiple species can be defined (in various shapes or point-wise). The case illustrated in Fig. 4 depicts a mouse source, namely a digimouse (Digimouse Atlas) [51,52], 

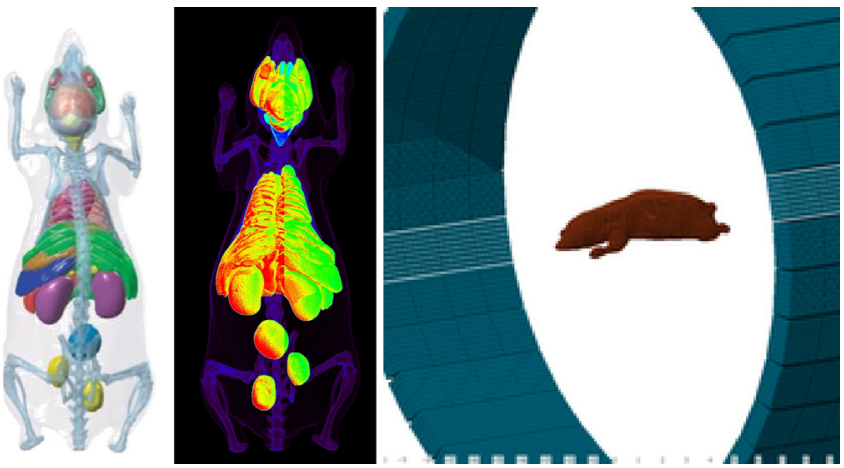

Fig. 4. On the left, a transversal view of the digimouse model [52], in the middle the PET image from a scanner and on the right the mouse geometry reconstructed in Flair placed in the detector model.

injected with FDG and placed in a MICROPET P4 [21]. In this case the radioisotope density map of the mouse is directly used as a source in FLUKA, converting the PET DICOM files.

\subsection{Image reconstruction}

As aforementioned, in addition to the already existing FBP reconstruction algorithm, a MLEM reconstruction code is now included, yielding higher image quality [47], as displayed in Fig. 5. For consistency, both the FBP and MLEM images were created with the same input, using $1 \times 10^{9}$ primary particles. The activity corresponded to $555 \mathrm{kBq}$ in an acquisition lasting $1800 \mathrm{~s}$.

While the FBP reconstruction time coincides with sinogram output generation, MLEM reconstructions require additional time. For the result depicted in Fig. 5, 70 iterations were employed, lasting approximately $4 \mathrm{~h}$.

\subsection{Applications to particle therapy}

As an example of in beam PET scenarios, the tools were applied to the reconstruction of PET signals proceeding from different ion beam irradiations, including $\beta^{+}$emitting radioactive ion beams, namely: ${ }^{11} \mathrm{C}$ $\left(t_{\frac{1}{2}} \approx 20 \mathrm{~min}\right),{ }^{12} \mathrm{C},{ }^{15} \mathrm{O}\left(t_{\frac{1}{2}} \approx 2 \mathrm{~min}\right)$ and ${ }^{16} \mathrm{O}$. The signal was collected according to both a realistic PET scanner and a voxelized patient geometry [24]. A Siemens Biograph $m \mathrm{cr}$ (TrueV version) scanner and beam delivery characteristics similar to that of the Heidelberg Ion Therapy
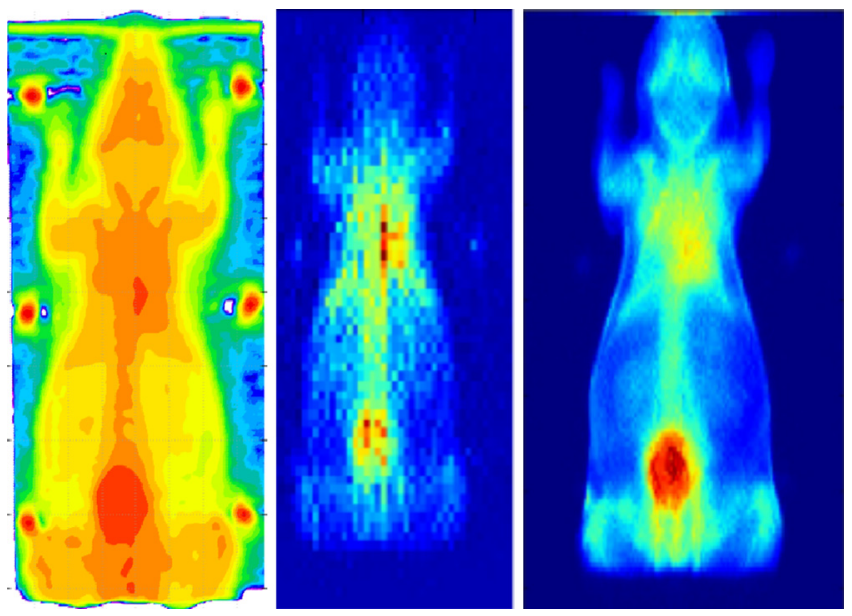

Fig. 5. On the left, the Flair visualization of the radioisotope density map in the mouse used as a source in FLUKA. In the subsequent images on the right, different methods for image reconstruction were applied, FBP is shown in the middle while the new MLEM method is shown on the right.
Center (HIT) were adopted. Furthermore, both an online and an offline PET signal acquisitions were simulated, employing an equivalent synchrotron-like irradiation with 16 spills with different energies (from the lowest to the highest) for each of the four beams under consideration. The resulting dose delivery reproduced a 3-cm long Spread Out Bragg Peak (SOBP) centered at $10 \mathrm{~cm}$ depth in an imported anthropomorphic head voxeL structure (centered at PET's FOV, as depicted in Fig. 6). The dose delivered corresponded to $1 \mathrm{~Gy}$ SOBPs. The calculation of beam energy was based on a fitting model devised for this purpose. On the other hand, the intensity for each pristine Bragg Peak was optimized with an algorithm package based on the FLUKA hadrontherapy framework [53]. Conversion factors from physical to biological dose were not employed in the presented simulations.

These simulations were intended as a demonstration of both the dosimetry and imaging properties of stable beams of oxygen and carbon ions compared to some of their radioactive counterparts $\left({ }^{11} \mathrm{C}\right.$ and $\left.{ }^{15} \mathrm{O}\right)$ in a clinical scenario. In particular, it was expected that an enhanced rate of annihilation event at rest, with the $\beta^{+}$emitting radioactive ion beam, would lead to a much improved PET image quality with comparable dose (Fig. 7).

Besides the enhancement of the annihilation events at rest, it was also expected to see a more direct correlation between the signal from radioactive ion beams and the SOBP dose profile, in range. Moreover, FLUKA PET TOOLS were expected to reflect the beam time structure, accounting for the evolution of $\beta^{+}$activity in time and the effects of different secondary radiation in the PET acquisition process. For all SOBPs, the irradiation took place during $130 \mathrm{~s}$, in 16 spills separated by $4 \mathrm{~s}$ pauses. The offline acquisition (Fig. 8) started $5 \mathrm{~min}$ after the irradiation end and lasted for $25 \mathrm{~min}$. In the presented configuration, the offline acquisition took place in the same room as the irradiation.

Online acquisition (Fig. 9) during the whole $130 \mathrm{~s}$ of irradiation (including pauses between the spills) time.

The reconstructed images of the SOBP irradiations' signal acquisition were obtained with the standard MLEM method available, employing 90 iterations which lasted a couple of minutes each to process the coincidence list and generate the images. Image reconstruction from either the coincidence list or sinogram output data can also be performed with external tools. One example of which, for the aforementioned SOBPs signal acquisition in sinogram format, is displayed in Figs. 10 and 11. Despite also being an iterative MLEM reconstruction code, it is optimized for the scanner model's actual FOV, with Fourier rebinning and intra-reconstruction smoothing [54,55].

The listing of various physical events can be requested by the user. By default, the user has access to the particle type, energy information (Fig. 12) and coordinates of every interaction in the PET scanner lattice structure, as well as the coordinates at which the radiation was produced. In the event of the radiation proceeding from a decay event, the parent isotope information is also available (see Fig. 13). For a $\beta^{+}$ emission this information is of great importance, in order to assess efficiency performance, verify the dominant isotopes at specific key times and analyze the count rate variation in time.

As mentioned in the description of the scoring routines, the user can obtain additional information by enabling the scoring of several extra quantities of interest making full use of FLUKA's capabilities.

Since the previously shown SOBPs were formed in layers from the lowest to the highest energy, the data collected was re-postprocessed to obtain a reverted SOBP layer delivery sequence. In this way the offline acquisition results would be penalized but the online acquisition would benefit from the contribution of the more relevant isoenergetic layers. The coincidence event counts' results obtained with a reverted SOBP are depicted in Fig. 14.

\section{Discussion}

The need for simulations in functional PET is inherently related to quantitative aspects (e.g. uptake value, target segmentation). This 

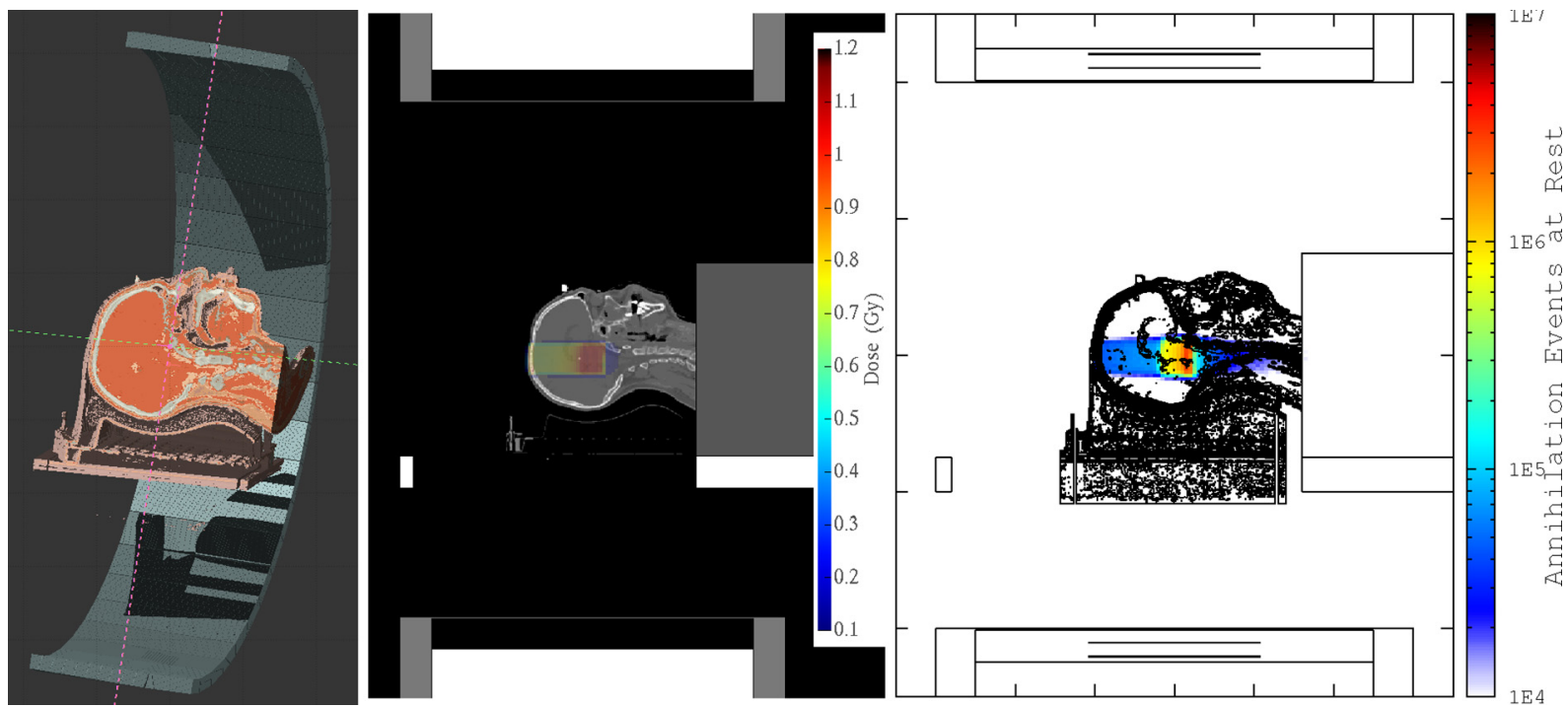

Fig. 6. 1 Gy SOBP irradiation with an ${ }^{15} \mathrm{O}$ beam (center), in an anthropomorphic voxelized geometry (left) imported into geoviewer and the total annihilation events at rest, time integrated (right).

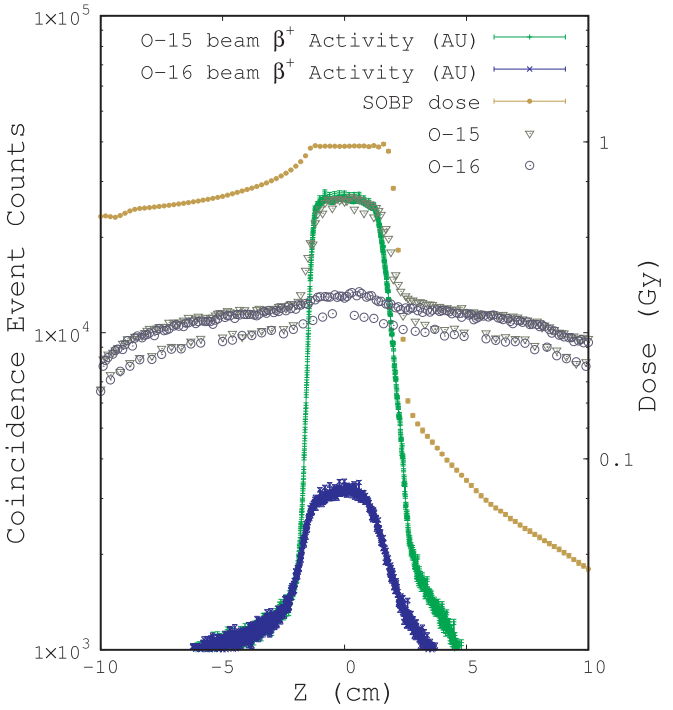

Oxygen ion beams (online)

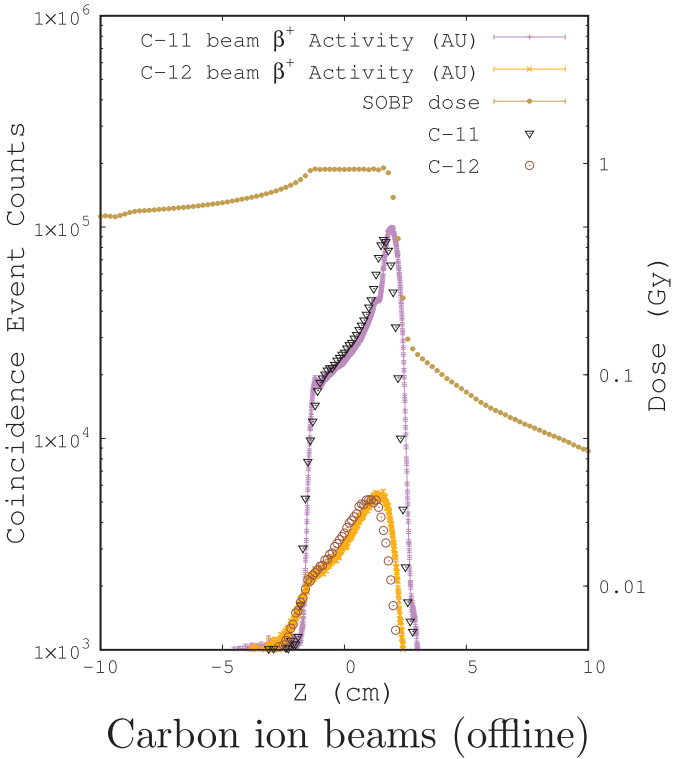

Carbon ion beams (offline)

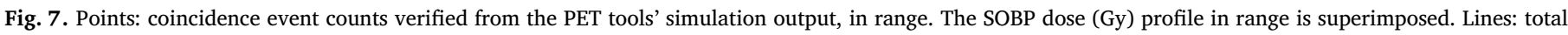

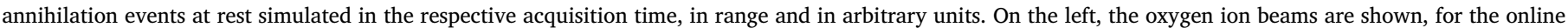
acquisition. On the right, only the carbon ion beams are shown, for the offline acquisition.

information is essential in diagnosis and eventually plays a major role in treatment planning for hadrontherapy. Hence, it evidently benefits from a higher detail in image reconstructions.

The MLEM algorithm now embedded in the FLUKA PET TOOLs, as shown in the mouse imaging example (Fig. 5), attains better quality than the FBP code included in the package's earlier version. However, it is important to stress that this is not intended as a comparison between the two techniques (their performance was not evaluated systematically), but instead between the results of the two reconstruction method versions implemented in the package. Moreover, even though the simulation input parameters were equal, the reconstruction using the MLEM algorithm required a much longer time (almost $4 \mathrm{~h}$ ) with respect to the FBP reconstruction.

Regarding the online and offline PET acquisitions, and irrespective of the methodology employed in the reconstruction, a (still rather qualitative) analysis over the images obtained is straightforward. In Fig. 7, the distal peak of the SOBP dose profile is observed at $1.63 \mathrm{~cm}$, the center of the SOBP corresponds approximately to the 0 position in the beam direction axis "Z" $(0.13 \mathrm{~cm})$. The $\mathrm{Z}$ origin is located at $10.0 \mathrm{~cm}$ in range from the patient's head beam entrance. The point in range at which the dose decreases to half of its peak value (50\% distal fall-off) is $2.18 \mathrm{~cm}$. By using radioactive ion beams, not only is the count rate approximately one order of magnitude higher than that observed with stable beams, one also sees a better correlation between the dose and coincidence event count's 50\% distal fall-offs. In fact, the 50\% distal fall-off for ${ }^{15} \mathrm{O}$ online coincidence event count occurs at $2.3 \mathrm{~cm}$, whereas ${ }^{16} \mathrm{O}$ is indistinguishable from the background. Please note that, since the SOBPs were formed by spills from the lowest energy layer to the highest, the online PET acquisition lacked some important signal contribution from the last energy layer. The distal edge would be more evident if the SOBP had been formed starting from the highest energy layer instead, or if the PET acquisition would have been extended in time. The former scenario was simulated in Fig. 14, and indeed reverted SOBPs of ${ }^{15} \mathrm{O}$ and ${ }^{11} \mathrm{C}$ ion beams resulted in much sharper distributions 

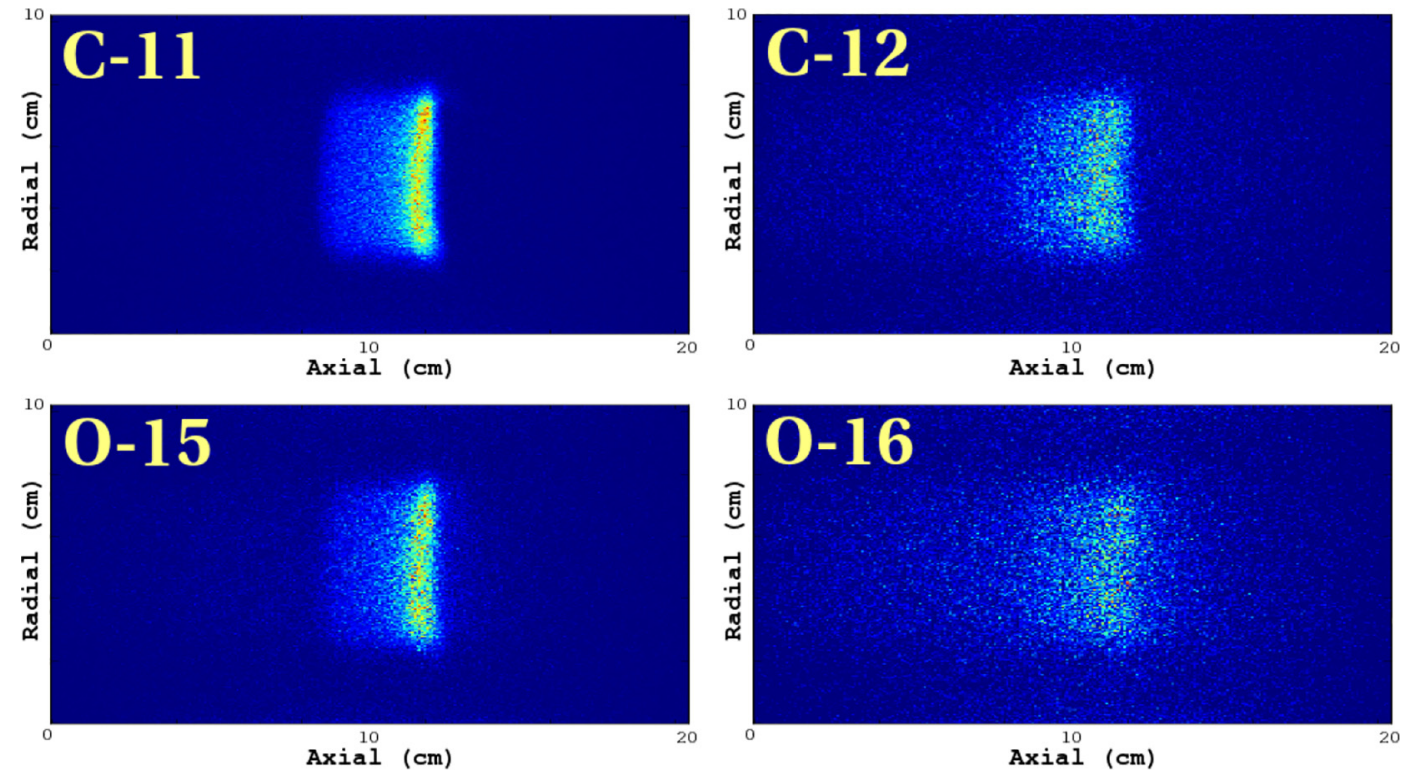

Fig. 8. Offline acquisition reconstruction for the four SOBPs studied using the total coincidences list, seen longitudinally. 90 iterations of the MLEM method were used.

of coincidence event counts, with $50 \%$ distal fall-off at 2.2 and $2.4 \mathrm{~cm}$, respectively. Also the ${ }^{15} \mathrm{O}$ gain in signal at peak value increased by over a factor of two using a reverted SOBP. As for the offline acquisition scenario, ${ }^{11} \mathrm{C}$ ion coincidence event count's $50 \%$ distal fall-off occurs at $2.1 \mathrm{~cm}$ in range, less than $1 \mathrm{~mm}$ from the SOBP dose profile distal fall-off. On the other hand ${ }^{12} \mathrm{C}$ ion coincidence event count's $50 \%$ distal fall-off takes place at $1.9 \mathrm{~cm}$ in range and its profile is much less sharp and not as well defined as for ${ }^{11} \mathrm{C}$.

In both image reconstruction methods used, one is able to discern the contour of the SOBPs, through signals from induced $\beta^{+}$emitter activity. The correlation between ${ }^{11} \mathrm{C}$ and ${ }^{15} \mathrm{O}$ signal with the SOBP dose distribution is also evident, particularly in range, along with an overall gain in image quality. Moreover, the more favorable distribution of $\beta^{+}$ activity in radioactive ( $\beta^{+}$emitting), over stable ion irradiations, is shown by its higher contrast and higher counts per pixel (Figs. 10 and 11). The acquisition time profile is also reflected on these results. The
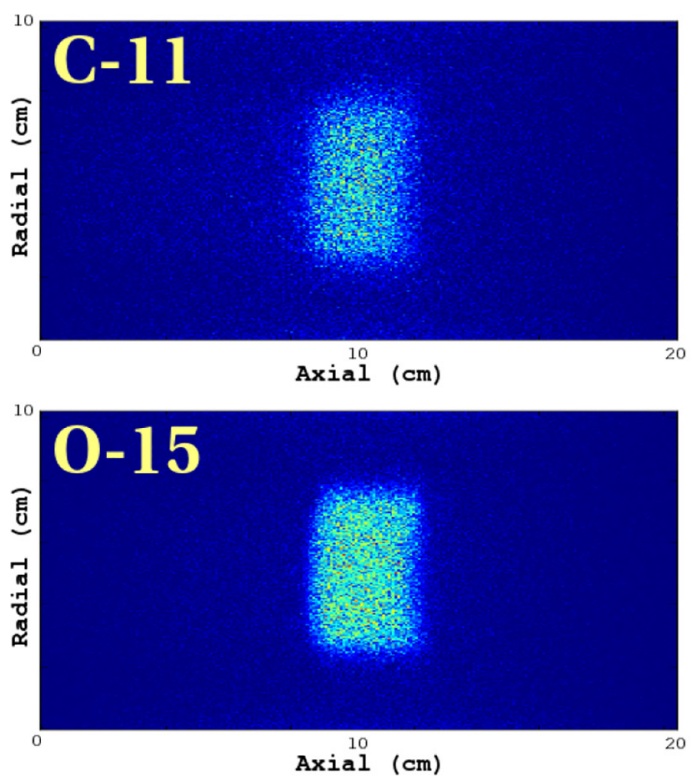

main $\beta^{+}$emitter contributing to the acquired signal in carbon and oxygen ion irradiation is ${ }^{11} \mathrm{C}$ and ${ }^{15} \mathrm{O}$, respectively. Due to their different half-life values, one can distinguish a considerably higher imaging quality for ${ }^{15} \mathrm{O}$ beam, which is short lived, during the $130 \mathrm{~s}$ of the online acquisition. Conversely, on the offline scenario, ${ }^{15} \mathrm{O}$ activity decreases substantially while ${ }^{11} \mathrm{C}$, in view of its longer half-life, attains an higher imaging quality performance. Although this is more evident for the radioactive ion beams' SOBPs, the same conclusions apply to a lesser degree to the stable ion beam irradiations considered as well. In Figs. 9 and 11 , since the irradiation took place from the lowest to the highest energy layer, the distal section of the SOBP is less noticeable on the online acquisition scenario as its contribution is almost unaccounted for. For the same reason, the distal peak is naturally highlighted in the offline (Figs. 8 and 10) acquisition scenario.

The simulations were able to reproduce the impact of the prompt mixed field in the image quality as well, which is reflected in the
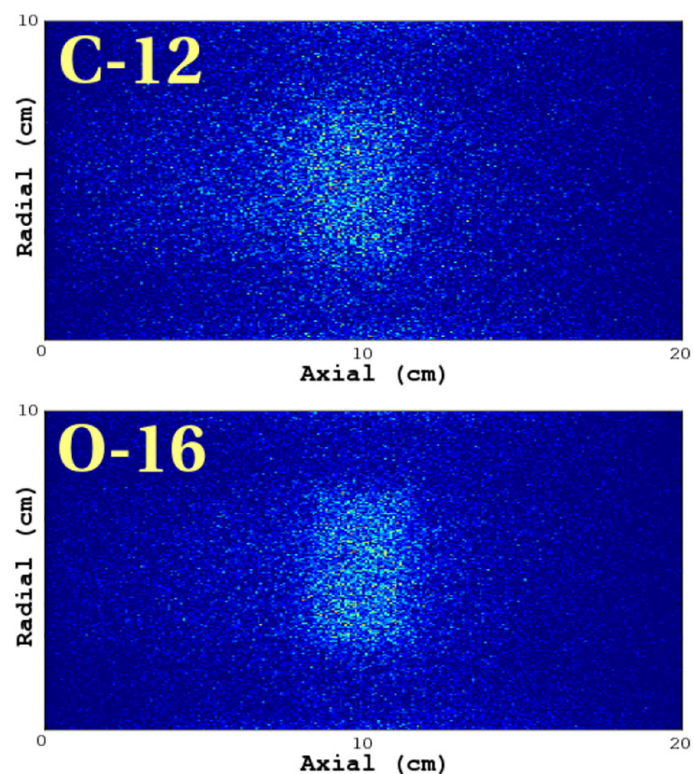

Fig. 9. Online acquisition reconstruction for the four SOBPs studied using the total coincidences list, seen longitudinally. 90 iterations of the MLEM method were applied. 

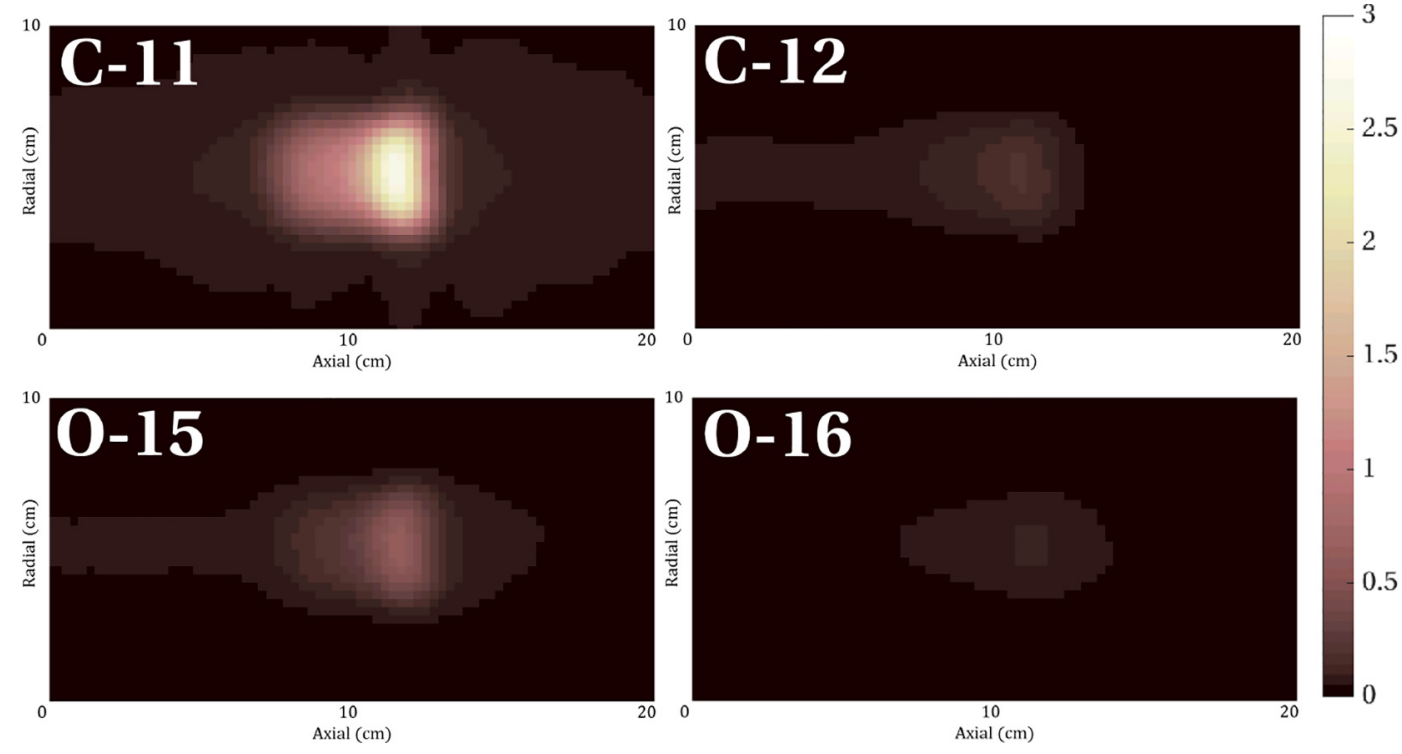

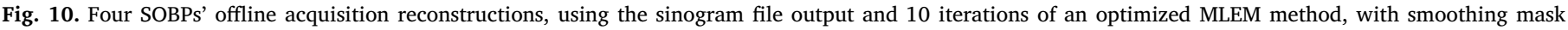
techniques and seen longitudinally.

general lower contrast of Fig. 11 with respect to the offline acquisitions. The possibility of disentangling the major isotope contributing to the coincidence event rate data collected in time from the overall signal, including the identification of the noise source, is shown in Fig. 13. This enables the user to thoroughly analyze the evolution of the count rate in time. It is possible to distinguish the higher relevance of ${ }^{8} \mathrm{~B}\left(t_{\frac{1}{2}} \approx 0.8 \mathrm{~s}\right)$ during spill whereas ${ }^{10} \mathrm{C}\left(t_{\frac{1}{2}} \approx 19 \mathrm{~s}\right)$ and ${ }^{15} \mathrm{O}$ contribution increases in between spills. Throughout the offline acquisition, it is observed that from an initially comparable $\beta^{+}$activity detected from ${ }^{15} \mathrm{O}$ and ${ }^{11} \mathrm{C}$ (over 1000 counts/s), the former's contribution disappears at the end whereas the latter's is still relatively high (500 counts/s).

Also, it was shown that the feasibility of an analysis of the coincidence event count distribution in energy (Fig. 12), by filtering the different events by parent isotope, allowed for an in-depth characterization of the acquired signal. In the aforementioned figures, it is clearly evident that the major $511 \mathrm{keV}$ line, the coincidence event count at that particular energy, is more than a factor of two higher for ${ }^{11} \mathrm{C}$ compared to ${ }^{12} \mathrm{C}$ ( $\sim 50,000$ counts contrarily to 20,000 during the $130 \mathrm{~s}$ of irradiation). For both species, one can also observe two lines, whose energies are correlated with the K-shell escape $\mathrm{x}$-ray emission from $\mathrm{Lu}$ (at approximately 450 and $457-458 \mathrm{keV}$ ), the major component of the scanner crystal material.

Presently, the tool is adapted for R\&D uses only as its use in clinical scenarios is still unfeasible in view of the long computational time required and the lack of attenuation corrections (and consequently lack of scatter correction as well). For the in beam PET calculations, 10 processors and approximately a week of CPU time is required, whereas for the conventional PET calculations, the simulations can be accomplished within a couple of hours. In this work a cluster of Intel Xeon processors (average of $2.4 \mathrm{GHz}$ ) was used. Either in an irradiation scenario or using a source distribution, the tools can be adapted to provide a coincidence list and general output containing relevant information to be used as feedback for PET scanner, treatment plan and beam line optimization.
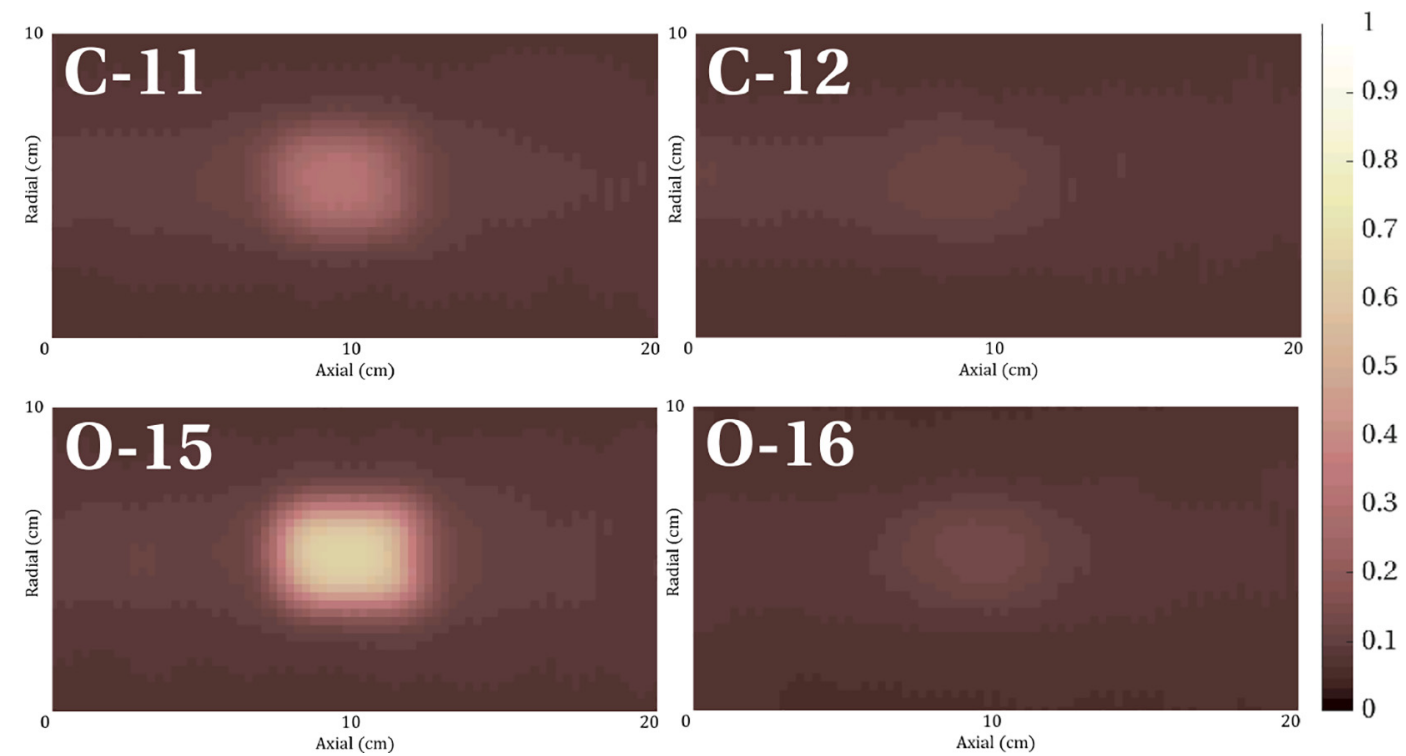

Fig. 11. Four SOBPs' online acquisition reconstructions, using the sinogram file output and 10 iterations of an optimized MLEM method, with smoothing mask techniques and seen longitudinally. 


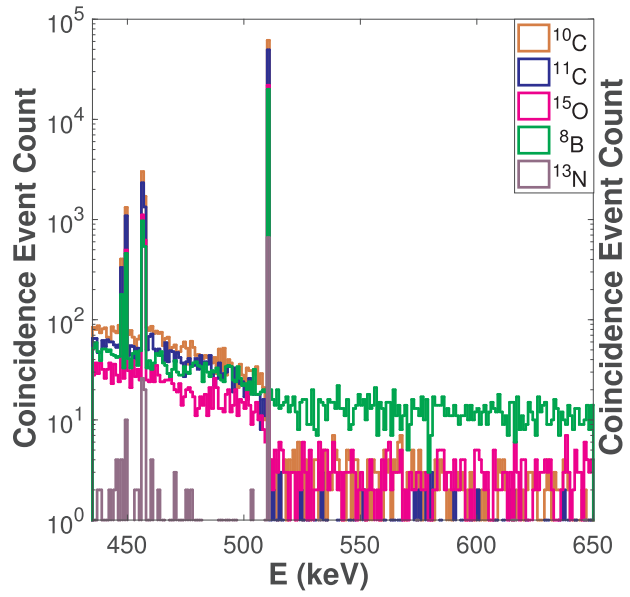

${ }^{11} \mathrm{C}$ SOBP

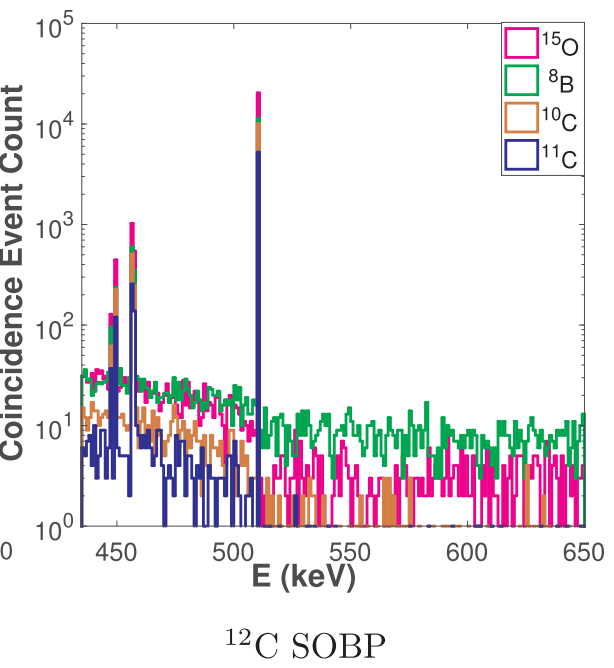

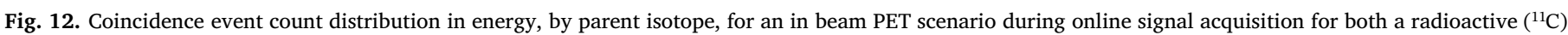

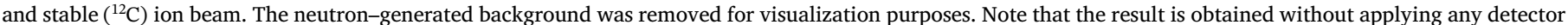
resolution.

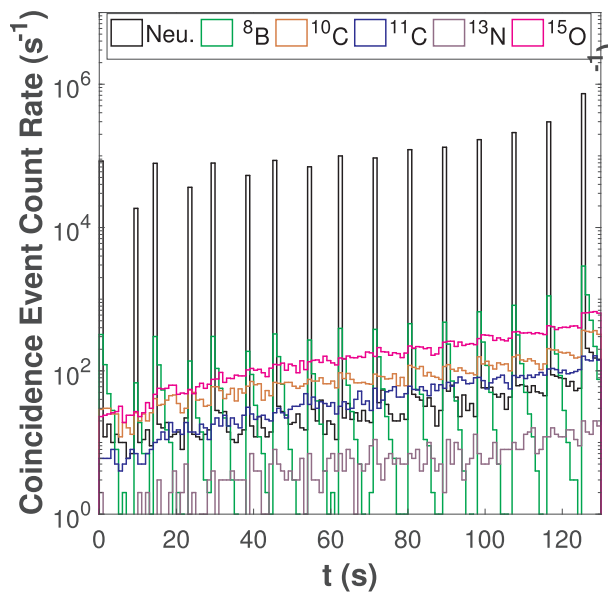

Online

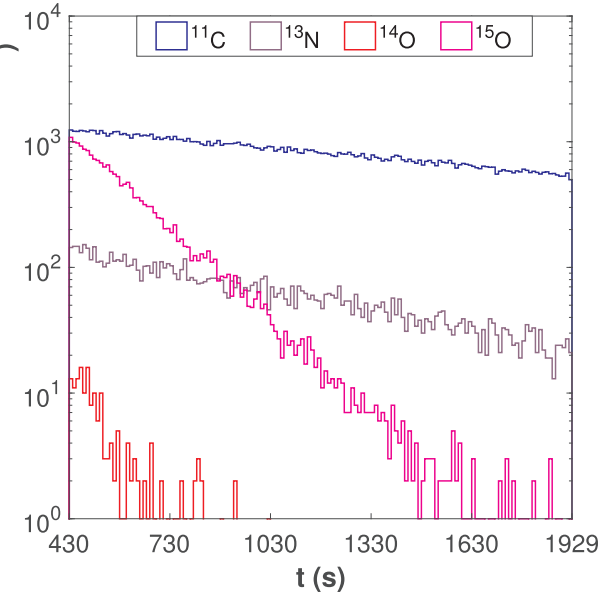

Offline

Fig. 13. Coincidence count rate evolution in time, by particle of origin, throughout the online and offline PET acquisition scenarios, for the ${ }^{12} \mathrm{C}$ SOBP.

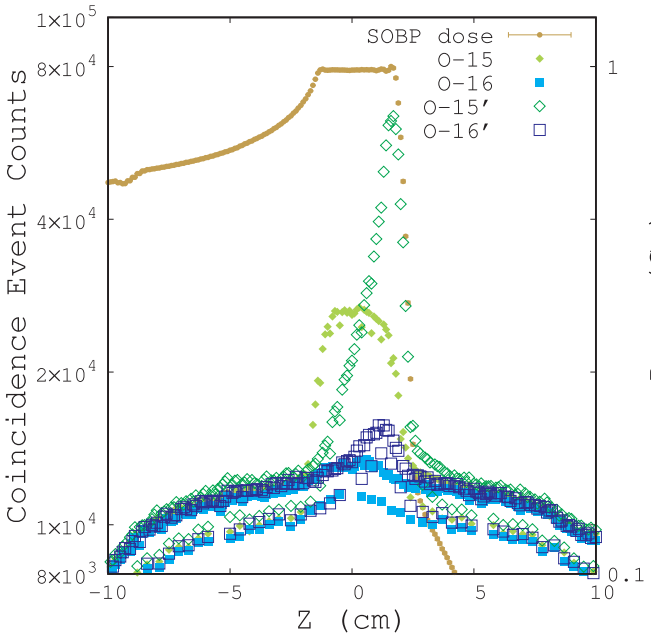

Oxygen ions

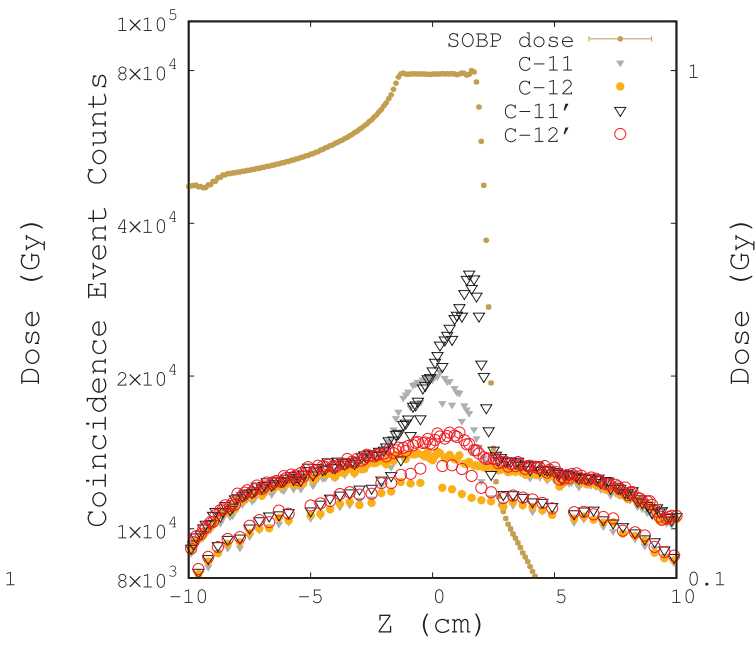

Carbon ions

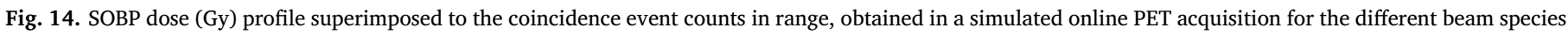
studied. The primed points denote coincidence events verified with a reverted SOBP. 


\section{Conclusion}

The tools hereby presented proved able of extending FLUKA's current capabilities towards PET reconstructions in complex scenarios, including acquisitions during beam irradiation and signal acquisition from intricate distributions of $\beta^{+}$emitters. Moreover, with its integration in FLUKA, it benefits from other options such as conversion of DICOM format to FLUKA voxeL geometry or hadrontherapy framework, allowing the user to adapt the tools to his/her needs, without additional packages and in an userfriendly manner.

In the presented work, the inclusion of extra options in the PET geometry building and processing phase were highlighted, along with the tools' optimized structure. Also, the new MLEM embedded reconstruction algorithm was shown to offer significant image quality improvement with respect to the previous FBP.

Ongoing work is aiming at including attenuation and scatter corrections. Regarding the future work, it will include: A quantitative analysis of the stable and radioactive ion beams' SOBP results in the framework of in beam PET image monitoring; Full validation of FLUKA PET Tools using National Electrical Manufacturers Association (NEMA) protocols and comparison with different codes; Testing of in beam PET scenarios in view of a possible simplification of the beam time/acquisition parameters still required to be inserted by the user; Assessment of possible clinical workflow integration and full integration in the FLUKA's Flair interface.

\section{Acknowledgements}

RS A and WS K acknowledge the financial support of the CERN-KT funding. C C sincerely thanks Prof. Ugo Amaldi for support and advice. P G O acknowledges financial support from Junta de Castilla y León and European ERDF funds under Contract No. SA041U16. The authors wish to thank Dr. Sofia Rollet and Dr. Aafke Kraan for testing the tools, as well as CNAO Medical Physics Team for providing the voxelized patient structure used in the simulation tests.

\section{References}

[1] Townsend DW. Positron emission tomography/computed tomography. Semin Nucl Med 2008;38(3):152-66.

[2] Ter-Pogossian MM, Phelps ME, Hoffman EJ, Mullani NA. A positron-emission transaxial tomograph for nuclear imaging (PETT). Radiology 1975;114(1):89-98.

[3] Zanzonico P. Positron emission tomography: a review of basic principles, scanner design and performance, and current systems. Semi Nucl Med 2004;34(2):87-111.

[4] Wilson RR. Radiological use of fast protons. Radiology 1946;47(5):487-91.

[5] Tobias C, Chatterjee A, Smith A. Radioactive fragmentation of N7+ ion beam observed in a beryllium target. Phys Lett A 1971;37(2):119-20.

[6] Parodi K. PET monitoring of hadrontherapy. Nucl Med Rev 2012;15(C):37-42.

[7] Pönisch F, Parodi K, Hasch BG, Enghardt W. The modelling of positron emitter production and PET imaging during carbon ion therapy. Phys Med Biol 2004;49(23):5217.

[8] Parodi K. On the feasibility of dose quantification with in-beam pet data in radiotherapy with ${ }^{12} \mathrm{C}$ and proton beams, PhD thesis, 2004.

[9] Min C-H, Kim CH, Youn M-Y, Kim J-W. Prompt gamma measurements for locating the dose falloff region in the proton therapy. Appl Phys Lett 2006;89(18):183517.

[10] Testa E, Bajard M, Chevallier M, Dauvergne D, Foulher FL, Freud N, et al. Monitoring the bragg peak location of $73 \mathrm{MeV} / \mathrm{u}$ carbon ions by means of prompt $\gamma$-ray measurements. Appl Phys Lett 2008;93(9):093506.

[11] Verburg JM, Seco J. Proton range verification through prompt gamma-ray spectroscopy. Phys Med Biol 2004;59(23):7089.

[12] Ortega PG, Torres-Espallardo I, Cerutti F, Ferrari A, Gillam JE, Lacasta C, et al. Noise evaluation of compton camera imaging for proton therapy. Phys Med Biol $1845 ; 60(5)$

[13] Parodi K. On- and off-line monitoring of ion beam treatment. Nucl Instrum Methods Phys Res Sect A: Accel, Spectrom, Detect Assoc Equip 2016;809(Suppl. C):113-9. advances in detectors and applications for medicine.

[14] Battistoni G, Collamati F, De Lucia E, Faccini R, Marafini M, Mattei I, et al. Design of a tracking device for on-line dose monitoring in hadrontherapy. Nucl Instrum Methods Phys Res Sect A: Accel, Spectrom, Detect Assoc Equip 2017;845:679-83.

[15] Muraro S, Battistoni G, Collamati F, De Lucia E, Faccini R, Ferroni F, et al. Monitoring of hadrontherapy treatments by means of charged particle detection. Front Oncol 2016;6:177.

[16] Chatterjee A, Alpen EL, Tobias CA, Llacer J, Alonso J. High energy beams of radioactive nuclei and their biomedical applications. Int J Radiat Oncol Biol Phys
1981;7(4):503-7

[17] Urakabe E, Kanai T, Kanazawa M, Kitagawa A, Noda K, Tomitani T, et al. Spot scanning using radioactive ${ }^{11} C$ beams for heavy-ion radiotherapy. Jpn J App Phys 2001;40(4R):2540.

[18] Mendonça T, Augusto R, Stora T. Intense post-accelerated ${ }^{11} C$ beams for hadrontherapy: Treatment and at the same time 3D dose mapping by PET imaging, CERN-ACC-NOTE-2014-0028.

[19] Augusto R, Mendonça T, Wenander F, Penescu L, Orecchia R, Parodi K, et al. New developments of ${ }^{11} C$ post-accelerated beams for hadron therapy and imaging, Nuclear Instruments and Methods in Physics Research Section B: Beam Interactions with Materials and Atoms 376 (Supplement C) (2016) 374-378, proceedings of the XVIIth International Conference on Electromagnetic Isotope Separators and Related Topics (EMIS2015), Grand Rapids, MI, U.S.A., 11-15 May 2015.

[20] Ortega PG, Böhlen TT, Cerutti F, Chin MPW, Ferrari A, Mairani A, et al. A dedicated tool for PET scanner simulations using FLUKA, 2013 3rd International Conference on Advancements in Nuclear Instrumentation, Measurement Methods and their Applications (ANIMMA) (2013) 1-7.

[21] Ortega PG, Böhlen TT, Cerutti F, Chin MPW, Ferrari A, Mancini C, et al. Development of a PET scanner simulation package for FLUKA. Radiother Oncol 2014;110:0167-8140.

[22] Ferrari A, Sala PR, Fasso A, Ranft J. FLUKA: a multi-particle transport code (Program version 2005), CERN-2005-010, SLAC-R-773, INFN-TC-05-11.

[23] Böhlen TT, Cerutti F, Chin MPW, Fassò A, Ferrari A, Ortega PG, et al. The FLUKA Code: Developments and Challenges for High Energy and Medical Applications. Nucl Data Sheets 2014;120:211-4.

[24] Battistoni G, Bauer J, Boehlen TT, Cerutti F, Chin MPW, Dos Santos Augusto R, et al. The FLUKA code: an accurate simulation tool for particle therapy. Front Oncol 2016;6:116.

[25] Vlachoudis V. Flair: a powerful but user friendly graphical interface for FLUKA. New York: Saratoga Springs; 2009.

[26] Böhlen TT, Ferrari A, Patera V, Sala PR. Describing Compton scattering and twoquanta positron annihilation based on Compton profiles: two models suited for the Monte Carlo method. J Instrum 2012;7(07):P07018.

[27] Parodi K, Enghardt W. Potential application of PET in quality assurance of proton therapy. Phys Med Biol 2000;45(11):N151.

[28] Parodi K, Enghardt W, Haberer T. In-beam PET measurements of $\beta^{+}$radioactivity induced by proton beams. Phys Med Biol 2002;47(1):21-36.

[29] Cerutti F, Dosanjh M, Ferrari A, Mendonça T, Ortega PG, Parodi K, et al. A systematic Monte Carlo study on the dosimetric and imaging properties of C-11 and O15 beams. Radiother Oncol 2016;118:0167-8140.

[30] Parodi K, Ponisch F, Enghardt W. The feasibility of in-beam PET for accurate monitoring of proton therapy: results of a comprehensive experimental study. IEEE Symp Conf Rec Nucl Sci 2004;2004(4):2160-4.

[31] Shakirin G, Braess H, Fiedler F, Kunath D, Laube K, Parodi K, et al. Implementation and workflow for PET monitoring of therapeutic ion irradiation: a comparison of inbeam, in-room, and off-line techniques. Phys Med Biol 2011;56(5):1281.

[32] Yamaya T, Inaniwa T, Minohara S, Yoshida E, Inadama N, Nishikido F, et al. A proposal of an open PET geometry. Phys Med Biol 2008;53(3):757.

[33] An SJ, Beak C-H, Lee K, Chung YH. A simulation study of a C-shaped in-beam PET system for dose verification in carbon ion therapy. Nucl Instrum Methods Phys Res Sect A: Accel, Spectrom, Detect Assoc Equip 2013;698(Suppl. C):37-43.

[34] Yamaya T. Openpet: a novel open-type PET system for 3D dose verification in particle therapy. J Phys: Conf Ser 2017;777(1):012023.

[35] Priegnitz M, Fiedler F, Kunath D, Laube K, Enghardt W. An experiment-based approach for predicting positron emitter distributions produced during therapeutic ion irradiation, IEEE Symposium Conference Record Nuclear Science 2012;59(1).

[36] Crespo P, Shakirin G, Enghardt W. On the detector arrangement for in-beam PET for hadron therapy monitoring. Phys Med Biol 2006;51(9):2143.

[37] Combs SE, Bauer J, Unholtz D, Kurz C, Welzel T, Habermehl D, et al. Monitoring of patients treated with particle therapy using positron-emission-tomography (PET): the MIRANDA study, in: BMC Cancer, 2012.

[38] Pawelke J, Byars L, Enghardt W, Fromm WD, Geissel H, Hasch BG, et al. Rossendorf Forschungszentrum, the investigation of different cameras for in-beam PET imaging. Phys Med Biol 1996;41(2). 279-279.

[39] Crespo P, Shakirin G, Fiedler F, Enghardt W, Wagner A. Direct time-of-flight for quantitative real-time in-beam PET: a concept and feasibility study. Phys Med Biol 2007;52(23):6795.

[40] Enghardt W, Crespo P, Fiedler F, Hinz R, Parodi K, Pawelke J, et al. Charged hadron tumour therapy monitoring by means of PET, Nucl Instrum Methods Phys Res Sect A: Accel, Spectrom, Detect Assoc Equip 2004;525(1):284-8, proceedings of the International Conference on Imaging Techniques in Subatomic Physics, Astrophysics, Medicine, Biology and Industry.

[41] Rosso V, Belcari N, Bisogni M, Camarlinghi N, Cirrone G, Collini F, et al. DoPET: an in-treatment monitoring system for proton therapy at $62 \mathrm{MeV}$. J Instrum 2006;11(12):C12029.

[42] Bisogni MG, Attili A, Battistoni G, Belcari N, Camarlinghi N, Cerello P, et al. INSIDE in-beam positron emission tomography system for particle range monitoring in hadrontherapy.

[43] Piliero MA, Pennazio F, Bisogni MG, Camarlinghi N, Cerello PG, Del Guerra A, et al. Full-beam performances of a PET detector with synchrotron therapeutic proton beams. Phys Med Biol 2016;61(23):N650-66.

[44] NEMA Organization, Digital Imaging and Communications in Medicine (DICOM) Standard, Tech. rep. (2014). URL http://dicom.nema.org.

[45] Schneider W, Bortfeld T, Schlegel W. Correlation between CT numbers and tissue parameters needed for Monte Carlo simulations of clinical dose distributions. Phys Med Biol 2000;45(2):459-78. 
[46] Defrise M, Kinahan PE, Townsend DW, Michel C, Sibomana M, Newport DF. Exact and approximate rebinning algorithms for 3-D PET data. IEEE Trans Med Imaging 1997;16(2):145-58.

[47] Toufique Y, A New Monte Carlo Tool to Simulate PET Scanner based on FLUKA, Qatar Foundation Annual Research Conference 2016;1(1).

[48] Todd JA, Cradduck T, Deconinck F. A file format for the exchange of nuclear medicine image data: a specification of Interfile version 3.3, Nucl. Med. Comm. 13 (1992) 673-699.

[49] Thielemans K, Tsoumpas C, Mustafovic S, Beisel T, Aguiar P, Dikaios N, et al. STIR: software for tomographic image reconstruction release 2. Phys Med Biol 2012;57(4):867. URL http://stacks.iop.org/0031-9155/57/i=4/a=867.

[50] Loening AM, Gambhir SS. AMIDE: a free software tool for multimodality medical image analysis. Mol Imaging 2003;2(3):131-7. http://dx.doi.org/10.1162/ 15353500200303133
[51] Stout D, Chow P, Silverman R, Leahy RM, Lewis X, Gambhir S, et al. Creating a whole body digital mouse atlas with PET, CT and cryosection images. Mol Imaging Biol 2002;4(4):S27.

[52] Dogdas B, Stout D, Chatziioannou AF, Leahy RM. Digimouse: a 3D whole body mouse atlas from CT and cryosection data. Phys Med Biol 2007;52(3):577.

[53] Kozłowska WS, Vlachoudis V. FLUKA Interface for Monte Carlo independent simulation for scanned proton and carbon ion beam therapy, to be published.

[54] Gianoli C, Kurz C, Riboldi M, Bauer J, Fontana G, Baroni G, et al. Clinical evaluation of 4D PET motion compensation strategies for treatment verification in ion beam therapy. Phys Med Biol 2016;61(11):4141.

[55] Gianoli C, Riboldi M, Kurz C, Bernardi ED, Bauer J, Fontana G, et al. PET-CT scanner characterization for PET raw data use in biomedical research. Computerized Med Imaging Graph 2014;38(5):358-68. 